\title{
Marshall-Peierls sign rules, the quantum Monte Carlo method, and frustration
}

\section{Document Version}

Accepted author manuscript

Link to publication record in Manchester Research Explorer

\section{Citation for published version (APA):}

Bishop, RF., \& Farnell, DJJ. (2000). Marshall-Peierls sign rules, the quantum Monte Carlo method, and frustration. In RF. Bishop, KA. Gernoth, NR. Walet, \& Y. Xian (Eds.), Recent Progress in Many-Body Theories: Proceedings of the 10th International Conference, Seattle, USA, September 10-15, 1999 (pp. 457-460). (Series on Advances in Quantum Many-Body Theory; Vol. 3). World Scientific Publishing Co. Pte. Ltd.

http://www.worldscibooks.com/physics/4434.html

\section{Published in:}

Recent Progress in Many-Body Theories

\section{Citing this paper}

Please note that where the full-text provided on Manchester Research Explorer is the Author Accepted Manuscript or Proof version this may differ from the final Published version. If citing, it is advised that you check and use the publisher's definitive version.

\section{General rights}

Copyright and moral rights for the publications made accessible in the Research Explorer are retained by the authors and/or other copyright owners and it is a condition of accessing publications that users recognise and abide by the legal requirements associated with these rights.

\section{Takedown policy}

If you believe that this document breaches copyright please refer to the University of Manchester's Takedown Procedures [http://man.ac.uk/04Y6Bo] or contact uml.scholarlycommunications@manchester.ac.uk providing relevant details, so we can investigate your claim.

\section{OPEN ACCESS}




\section{Series on Advances in Quantum Many-Body Theory - Vol. 3}

The Proceedings of the 10th International Conference

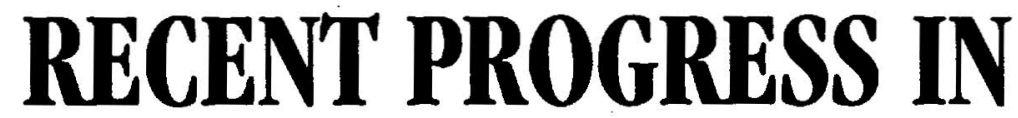
MANY-BODY THEORIES

Seattle, USA September $10-15,1999$

Editors

Raymond F. Bishop

Klaus A. Gernoth

Niels R. Walet

Yang Xian

UMIST, Manchester, UK 


\section{Published by}

World Scientific Publishing Co. Pte. Ltd.

P O Box 128, Farrer Road, Singapore 912805

USA office: Suite 1B, 1060 Main Street, River Edge, NJ 07661

UK office: 57 Shelton Street, Covent Garden, London WC2H 9HE

British Library Cataloguing-in-Publication Data

A catalogue record for this book is available from the British Library.

\section{RECENT PROGRESS IN MANY-BODY THEORIES (RPMBT-10)}

Copyright $\odot 2000$ by World Scientific Publishing Co. Pte. Ltd.

All rights reserved. This book, or parts thereof, may not be reproduced in any form or by any means, electronic or mechanical, including photocopying, recording or any information storage and retrieval system now known or to be invented, without written permission from the Publisher.

For photocopying of material in this volume, please pay a copying fee through the Copyright Clearance Center, Inc., 222 Rosewood Drive, Danvers, MA 01923, USA. In this case permission to photocopy is not required from the publisher.

ISBN 981-02-4318-9

Printed in Singapore by Uto-Print 


\title{
MARSHALL-PEIERLS SIGN RULES, THE QUANTUM MONTE CARLO METHOD, AND FRUSTRATION
}

\author{
R. F. BISHOP AND D. J. J. FARNELL
}

Department of Physics, UMIST, P.O. Box 88, Manchester M60 1QD, United Kingdom.

In $1955 \mathrm{Marshall}^{1}$ used a variational method to study the isotropic spin-half Heisenberg antiferromagnet (HAF) specified by the Hamiltonian

$$
H=J \sum_{\langle i, j\rangle} \mathbf{s}_{i} \cdot \mathbf{s}_{j}
$$

where the sum on $\langle i, j\rangle$ counts each nearest-neighbour pair once and once only, and $J$ is positive. Furthermore, the lattice was assumed to be bipartite (i.e., the lattice can be divided into two sublattices $(A, B)$ such that all nearest neighbours of a site on one sublattice lie on the other and vice versa). The total number of atoms was $N$ and the spatial dimensionality was not restricted. In the course of this paper it was proven that the exact ground-state wave function can be written as

$$
|\Psi\rangle=\sum_{I} c_{I}|I\rangle
$$

where $\{|I\rangle\}$ are the usual basis states in the Ising representation. The coefficients $\left\{c_{I}\right\}$ were proven to have the property that, $c_{I}=(-1)^{\phi(I)} a_{I}$, where the coefficients $\left\{a_{I}\right\}$ are all positive real numbers or zero, and $\phi(I)$ is the eigenvalue, with respect to the corresponding eigenstate $|I\rangle$, of the operator, $\phi=n_{A}$, which counts all of the spin-half 'up' states on the $A$-sublattice. Lieb and Mattis ${ }^{2}$ proved that the ground state of the HAF was a singlet, following the work of Marshall ${ }^{1}$ and Lieb et al. ${ }^{3}$

Indeed, these results for the HAF model on a bipartite lattice are a consequence of a property of non-positive matrices. The Hamiltonian of Eq. (1) is transformed in the following manner,

$$
H^{\prime}=e^{i \pi \phi} H e^{-i \pi \phi}
$$

It is seen that $H^{\prime}$ of Eq. (3) contains only non-positive off-diagonal interactions with respect to the Ising basis states. Hence the ground state of the HAF corresponds to the eigenstate of largest magnitude eigenvalue which is positive-definite via the Perron-Frobenius theorem. ${ }^{4}$ Munro $^{5}$ and Parkinson ${ }^{6}$ were also able to extend these results to the spin-one biquadratic model on a bipartite lattice in various phases. They showed that the ground eigenstates are positive-definite and also non-degenerate. Finally, Klein ${ }^{7}$ utilized this knowledge afforded by the PerronFrobenius theorem for Heisenberg models to prove six theorems relating to groundstate features of these models. Amongst these theorems was one which stated that the ground-state expectation value of $\mathbf{s}_{i} \cdot \mathbf{s}_{j}$ for these models is positive or negative depending on whether $i$ and $j$ are on the same or different sublattices, respectively. We now attempt to extend these earlier results to other models. The first model 
that we consider is the $X Y Z$ model, which has a Hamiltonian given by

$$
\begin{aligned}
H_{X Y Z} & =\sum_{[i, j]}\left\{J_{i, j}^{x} s_{i}^{x} s_{j}^{x}+J_{i, j}^{y} s_{i}^{y} s_{j}^{y}+J_{i, j}^{z} s_{i}^{z} s_{j}^{z}\right\} \\
& =\sum_{[i, j]}\left\{a_{i, j} s_{i}^{z} s_{j}^{z}+b_{i, j}\left(s_{i}^{+} s_{j}^{-}+s_{i}^{-} s_{j}^{+}\right)+c_{i, j}\left(s_{i}^{+} s_{j}^{+}+s_{i}^{-} s_{j}^{-}\right)\right\}
\end{aligned}
$$

where $a_{i, j}=J_{i, j}^{z}, b_{i, j}=\left(J_{i, j}^{x}+J_{i, j}^{y}\right) / 4$ and $c=\left(J_{i, j}^{x}-J_{i, j}^{y}\right) / 4$. The index $i$ in Eq. (4) runs over all $N$ sites on a bipartite lattice and $j$ runs over all sites which are on the opposite sublattice to $i$. The square bracket $[i, j]$ indicates that each bond is counted once and once only. The second such model that we shall consider is the single-ion anisotropy (SIA) model, given by

$$
\begin{aligned}
H_{\mathrm{SIA}} & =\sum_{[i, j]} J_{i, j}\left\{s_{i}^{z} s_{j}^{z}+\frac{1}{2}\left(s_{i}^{+} s_{j}^{-}+s_{i}^{-} s_{j}^{+}\right)\right\} \\
& +\frac{D}{4} \sum_{i}\left\{\left(s_{i}^{+}\right)^{2}+\left(s_{i}^{-}\right)^{2}+2\left(s(s+1)-\left(s_{i}^{z}\right)^{2}\right)\right\} .
\end{aligned}
$$

Again, the index $i$ in Eq. (5) runs over all $N$ sites on a bipartite lattice and $j$ runs over all sites which are on the opposite sublattice to $i$. Finally, we also consider the transverse Ising model (TIM), which has a Hamiltonian given by

$$
H_{\mathrm{TIM}}=\sum_{\left[k_{1}, k_{2}\right]} \Omega_{k_{1}, k_{2}} s_{k_{1}}^{z} s_{k_{2}}^{z}+\frac{\lambda}{2} \sum_{i}\left(s_{i}^{+}+s_{i}^{-}\right) .
$$

For the TIM, we place no restriction on the lattice type, and $k_{1}$ and $k_{1}$ are allowed to run over all lattice sites with $k_{1} \neq k_{2}$. Furthermore, note that adding extra diagonal terms in Eqs. (4-6) (such as an external magnetic field or crystal field anisotropy in the $z$-direction), or letting $i$ and $j$ in Eqs. (4) and (5) run over the same sublattice but, in this case, explicitly restricting $b_{i, j} \leq 0$ in Eq. (4) and $J_{i, j} \leq 0$ in Eq. (5), do not change the following sign rules.

We now define the following operators,

$$
m_{A}=\sum_{l_{A}} s_{l_{A}}^{z} ; m=\sum_{l} s_{l}^{z}
$$

where $l_{A}$ runs over all $N / 2 A$-sublattice sites and $l$ runs over all $N$ sites on both sublattices. Note that for eigenstate $|I\rangle=\bigotimes_{l=1}^{N}\left|s_{l}, m_{l}\right\rangle$, where $s_{l}^{z}\left|s_{l}, m_{l}\right\rangle=m_{l}\left|s_{l}, m_{l}\right\rangle$, $m$ and $m_{A}$ have eigenvalues denoted by $m(I)$ and $m_{A}(I)$, respectively. Note that the following sign rules are fully defined by the form of the operator $\phi$ which, in turn, gives the set of eigenvalues $\{\phi(I)\}$. Furthermore, these eigenvalues determine the signs of the $\left\{c_{I}\right\}$ coefficients for the expansion of the ground-state wave functions, Eq. (2), of the anisotropic Hamiltonians of Eqs. (4-6). The sign rules are 
thus defined by,

$$
\left.\begin{array}{lll}
\phi=1 & ; & \text { rule (A) } \\
\phi=m_{A} & ; & \text { rule (B) } \\
\phi=m / 2 & ; \operatorname{rule~(C)~} \\
\phi=m_{A}+m / 2 & ; \text { rule (D) } \\
\phi=m & ; \text { rule (E) }
\end{array}\right\}
$$

Furthermore, note that rule (B) is a reformulation, for general spin quantum number, of the Marshall-Peierls sign rule for the spin-half HAF. It is found that the first four sign rules apply to the $X Y Z$ and SIA models in the following regimes,

$\left.\begin{array}{ccc}\text { Rule } & X Y Z \text { model } & \text { SIA model } \\ \text { (A) } & b_{i, j} \leq 0, c_{i, j} \leq 0 & D \leq 0, J_{i, j} \leq 0 \\ \text { (B) } & b_{i, j}>0, c_{i, j} \geq 0 & D \leq 0, J_{i, j}>0 \\ \text { (C) } & b_{i, j} \leq 0, c_{i, j}>0 & D>0, J_{i, j} \leq 0 \\ \text { (D) } & b_{i, j}>0, c_{i, j}<0 & D>0, J_{i, j}>0\end{array}\right\}$

for all values of $i$ and $j$ on opposite sublattices. (Note again that we do not allow the existence of any "frustrated" interactions in Eqs. (4) or (5).) For the TIM, it is found that rule (A) applies when $\lambda \leq 0$, and rule (E) applies when $\lambda>0$ for all signs and strengths of the coefficients $\Omega_{k_{1}, k_{2}}$ of the Ising interaction in Eq. (6). The effect of these sign rules and the transformation of Eq. (3) is now that,

$$
\left\langle I_{1}\left|\left(H^{\prime}-w\right)\right| I_{2}\right\rangle \leq 0 \quad \forall I_{1}, I_{2} ;
$$

where $w$ is a positive number large enough to force the diagonal elements to be always negative. Note that the following relationship also holds:

$$
\left\langle I_{1}\left|\exp \left[\alpha\left(H^{\prime}-w\right)\right] .\right| I_{2}\right\rangle \geq 0 \forall I_{1}, I_{2} ; \text { if } \alpha \leq 0 \text {. }
$$

The of $H$ may be written as, $|\Psi\rangle=\sum_{I} \exp [-\mathrm{i} \pi \phi(I)] a_{I}|I\rangle$, where $a_{I} \geq 0 \forall I$. We note that in all cases, although the individual phase factors $\exp [-\mathrm{i} \pi \phi(I)]$ may be complex, the relative phase factors are always real in the specified regimes.

We now turn our attention to quantum Monte Carlo (QMC) calculations. All QMC methods at zero temperature basically project the exact ground state $|\Psi\rangle$ of a given many-body Hamiltonian $H$ out of an initial trial state $|\Phi\rangle$ not orthogonal to $|\Psi\rangle$, by repeated applications of some suitable projection operator, $G=G(H)$. This operation can be formally expressed as a path integral over many-particle trajectories in configuration space. Furthermore, it can thus be represented by a stochastic process, which may itself be simulated computationally by the random walks of a sampled set of independent "walkers" through the configuration space. The main limitation to the applicability of such QMC techniques arises from the positivity requirement, $p\left(I, I^{\prime}\right) \equiv\left\langle I|G| I^{\prime}\right\rangle \Phi\left(I^{\prime}\right) / \Phi(I)>0$, on the probability $p\left(I, I^{\prime}\right)$ for a walker to make a transition from an initial configuration $|I\rangle$ to another configuration $\left|I^{\prime}\right\rangle$ along a random walk, where $\Phi(I)$ is the wave function of the initial trial state. If we choose $G \equiv \exp \left[-\tau\left(H^{\prime}-w\right)\right]$, with $\tau \geq 0$ and $\Phi(I) \geq 0 \forall I$, we may see from Eq. (11) that this is indeed satisfied.

Finally, we note that sign rules are difficult to prove when off-diagonal terms in the Hamiltonian compete against each other (i.e., frustration). However, one may 


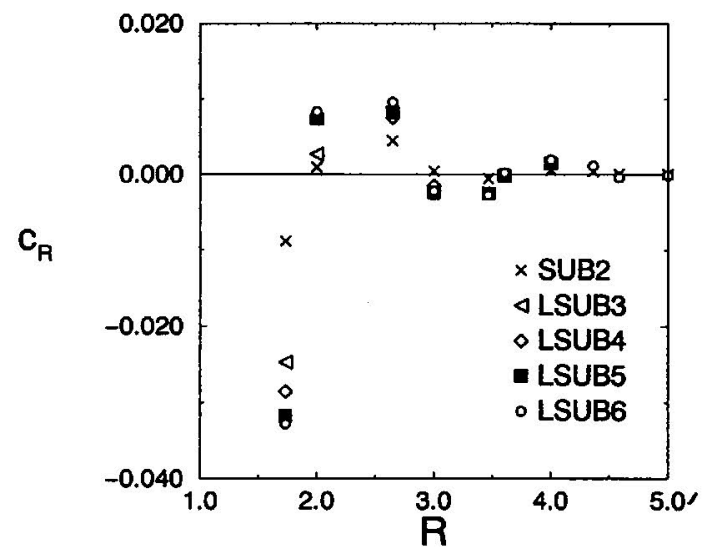

Figure 1. Ising expansion coefficients corresponding to two-body excitations plotted against lattice distance, $R$, for the triangular lattice HAF using the CCM technique.

use approximate methods, such as the coupled cluster method ${ }^{8}$ (CCM), in order to probe the nodal surface in the Ising basis. The CCM ground-state ket wave function is given by,

$$
|\Psi\rangle=e^{S}|\Phi\rangle
$$

where $|\Phi\rangle$ is the model state. Eqqs. (2) and (12) are used in order to obtain approximate values for $\left\{c_{I}\right\}$. Fig. 1 plots these for two-body excitations with respect to $|\Phi\rangle$ as a function of the lattice distance, $R$, for the spin-half Heisenberg antiferromagnet (HAF) on the triangular lattice, for which exact results are not known. The LSUB $m$ approximation, which includes all $m$-body (or lower-order) correlations in $S$ in a locale also defined by $m$, and the SUB2 approximation, which includes all two-body correlations in $S$ but with no further restriction on the lattice distance, are utilised in these calculations. ${ }^{8}$ Hence, the CCM is simulating from an $a b$ initio point-of-view the nodal surface of this frustrated spin model.

\section{References}

1. W. Marshall, Proc. Roy. Soc. A 232, 48 (1955).

2. E. H. Lieb and D. C. Mattis, J. Math. Phys. 3, 749 (1962).

3. E. H. Lieb, T. D. Schultz, and D. C. Mattis, Ann. Phys. (N. Y.) 16, 407 (1961).

4. O. Perron, Math. Ann. 64, 248 (1907); G. Frobenius, Sitzungsber. Preuss. Akad. Wiss. (Berlin) Math.-Nat. Kl. 471 (1908); R. A. Horn and C. R. Johnson, Matrix Analysis (Cambridge University Press, Cambridge, 1985).

5. R. G. Munro, Phys. Rev. B 13, 4875 (1976).

6. J. B. Parkinson, J. Phys. C: Solid State Phys. 10, 1735 (1977).

7. D. J. Klein, J. Chem. Phys. 77, 3098 (1982).

8. R. F. Bishop, D. J. J. Farnell, and Chen Zeng, Phys. Rev. B 59, 1000 (1999). 